\title{
Perspective
}

PERSPECTIVE Actualité en histoire de l'art

$1 \mid 2020$

Japon

\section{Le jardin japonais comme champ des enjeux internationaux : tendances récentes de la recherche}

The Japanese Garden as a Field of International Issues: Recent Research Trends

Der japanische Garten als Themengebiet internationaler Fragestellungen:

Neueste Forschungstrends

Il giardino giapponese come campo di sfide internazionali: tendenze recenti della ricerca

El jardín japonés como el campo de las problemáticas internacionales:

tendencias recientes de las investigaciones

\section{Hiromi Matsugi}

\section{OpenEdition}

Journals

Édition électronique

URL : http://journals.openedition.org/perspective/18948

DOI : 10.4000/perspective.18948

ISSN : 2269-7721

Éditeur

Institut national d'histoire de l'art

Édition imprimée

Date de publication : 5 juin 2020

Pagination : 257-266

ISBN : 978-2-917902-89-9

ISSN : $1777-7852$

\section{Référence électronique}

Hiromi Matsugi, «Le jardin japonais comme champ des enjeux internationaux : tendances récentes de la recherche », Perspective [En ligne], 1 | 2020, mis en ligne le 30 décembre 2020, consulté le 25 janvier 2021. URL : http://journals.openedition.org/perspective/18948; DOI : https://doi.org/10.4000/ perspective. 18948 


\title{
Le jardin japonais comme champ des enjeux internationaux : tendances récentes de la recherche
}

\author{
Hiromi Matsugi
}

La popularité internationale du jardin japonais est incontestable, quand on observe le nombre de touristes affluant vers les temples et les palais de l'ancienne capitale Kyōto, et la parution de livres en langues étrangères qui donnent des instructions pour créer son propre jardin japonais chez soi. En effet, parmi les multiples facettes de la société japonaise, le jardin japonais cristallise ce qui est attractif dans sa culture, c'est-à-dire sa capacité à offrir des remèdes au monde moderne oppressant : la relation harmonieuse avec la nature, et la sérénité d'âme. Si les recherches scientifiques, malgré leurs efforts pour parvenir à une vision plus objective, n'ont pas le pouvoir de modifier cette image populaire, c'est probablement parce que le jardin est considéré avant tout comme une création endogène, intrinsèquement liée à l'environnement naturel de la région où il se trouve. Cette conception du jardin sous-tend non seulement l'appréciation positive du jardin japonais comme œuvre ancrée dans un territoire ancestral, mais elle semble aussi avoir enfermé le champ d'études à l'intérieur des frontières nationales et empêché d'apercevoir sa dimension internationale.

Cet article présente, à travers la lecture de quelques ouvrages et articles, deux tendances émergeant depuis environ trois décennies qui renouvellent les approches dans les recherches universitaires, en abordant le jardin japonais comme champ des enjeux internationaux. La première tendance est celle qui examine le mécanisme complexe de la perception, de la réflexion et de la circulation d'idées entre les acteurs japonais et étrangers, qui engendre certaines images essentialistes du jardin japonais et le mythe du génie national qui y est associé. La deuxième est celle qui prend comme objet d'étude les jardins japonais créés en dehors du Japon, pour interroger les valeurs du jardin japonais dans des contextes de création internationaux, là où ne s'applique plus la logique d'un lien intrinsèque entre l'homme et son environnement naturel ancestral.

\section{Déconstruction des images du jardin japonais et du mythe du génie national}

Comment une image du jardin japonais est-elle née et a-t-elle été remplacée par une autre dans un va-et-vient des discours entre l'Occident et le Japon ? Tel est le sujet 


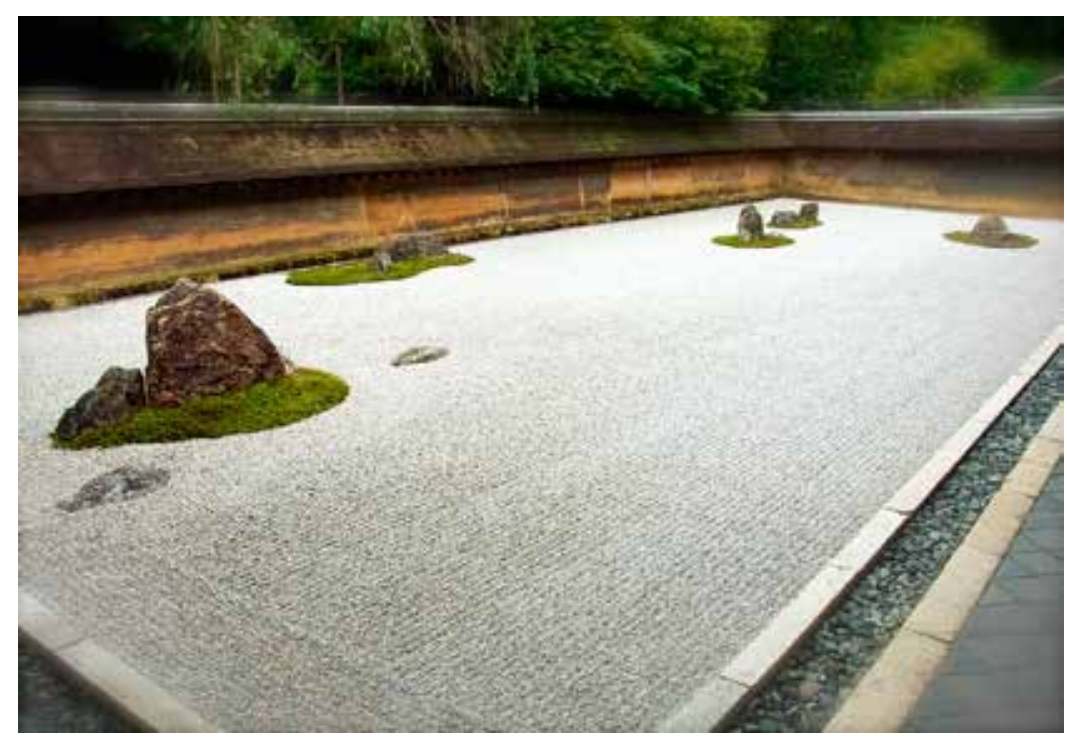

1. Jardin du temple Ryōan-ji, Kyōto. d'étude de Nihon teien zō no keisei [La formation de l'image du jardin japonais], ouvrage de Miyuki Katahira, basé sur sa thèse de doctorat de 2004, traitant cette question durant une période cruciale allant des années 1880 aux années $1930^{1}$. Katahira analyse d'abord quelques ouvrages de la fin du XIX ${ }^{e}$ siècle qui sont devenus des références incontournables en Occident, tels que Landscape Gardening in Japan de Josiah Conder (1893), Japanese Homes and their Surroundings d'Edward Morse (1886), et Glimpse of Unfamiliar Japan (1894) de Lafcadio Hearn. Elle explique l'appréciation positive des jardins japonais chez ces expatriés et voyageurs anglais et américains par le contexte de la recherche du naturel dans les jardins de leurs propres pays ; ces auteurs trouvaient, en effet, plus naturels et harmonieux les jardins du Japon que les jardins réguliers, dits à la française, ou les jardins paysagers, appelés aussi à l'anglaise, dont la géométrie, l'asymétrie, ou l'accumulation des fabriques empruntées à des civilisations et à des époques disparates ont été critiquées comme excessivement artificielles.

Katahira démontre ensuite comment cette image du jardin japonais, basée sur l'observation des jardins ordinaires et la lecture des manuels de l'époque d'Edo (XVII ${ }^{\mathrm{e}}$-XIX ${ }^{\mathrm{e}}$ siècle), a cédé, dans les années 1920 et 1930, devant celle plus spirituelle et austère, représentée par les jardins secs de temples zen à Kyōto. Ces jardins de l'époque de Muromachi (XIVe $\mathrm{XVI}^{\mathrm{e}}$ siècles), caractérisés par des rochers posés sur un tapis de sable ratissé, ont été mis en valeur par Jirō Harada, auteur de The Gardens of Japan (1928), puis par d'autres spécialistes et intellectuels japonais, comme Shōkichi Harigaya, Tsuneyoshi Tsuzumi, Mirei Shigemori, Tsuyoshi Tamura et Matsunosuke Tatsui, qui éprouvaient la nécessité de faire évaluer les jardins japonais par les Japonais mêmes. Katahira explique cette tendance collective par la prise de conscience du regard occidental, l'institutionnalisation des études sur le jardin en tant que discipline universitaire au Japon, et surtout la volonté de singulariser la culture japonaise dans un climat nationaliste général à la veille des grands conflits militaires. Le jardin japonais incarne désormais, avec l'art floral et la cérémonie du thé, l'esthétique du raffinement dans la simplicité, le génie national qui dépasse à la fois l'art occidental et l'art oriental. Cette nouvelle image du jardin japonais, ainsi que la vision historique de l'art du jardin avec son apogée à la période de Muromachi et son déclin 
à la période d'Edo, ont été largement repris, conclut-elle, dans les ouvrages occidentaux, à partir de One Hundred Kyōto Gardens de Loraine Kuck (1935).

La déconstruction de l'image du jardin japonais est également l'objectif de l'ouvrage de Shōji Yamada, Zen to yū na no nihonmaru, traduit en anglais sous le titre de Shots in the Dark: Japan, Zen, and the West ${ }^{2}$. Il choisit comme cas d'étude le jardin du temple Ryōan-ji à Kyōto (fig. 1), qui est sans doute le jardin japonais le plus célèbre aujourd'hui dans le monde, afin d'analyser le processus et le mécanisme selon lesquels ce jardin est parvenu à représenter la culture japonaise en général. Chercheur en informatique en sciences humaines, Yamada effectue la relecture d'une large gamme de textes historiques, en allant des commentaires des spécialistes du jardin aux manuels d'histoire nationale, en passant par des essais d'écrivains, pour observer une évolution du discours entre les années 1920 et les années 1960, occasionnée souvent par des interprétations forcées, qui aboutit à la mythification et à l'iconisation de ce jardin. Le jardin de Ryōan-ji, composé de quinze pierres posées sur le sable ratissé, avait été considéré, jusqu'à la fin du XIX ${ }^{e}$ siècle, comme la visualisation d'une légende chinoise sur le tigre traversant la rivière avec ses enfants sur le dos. Les avis divergeaient cependant sur sa qualité esthétique, pour deux raisons principalement : son concepteur demeurait non identifié et son apparence changeait - parfois, on y notait la présence d'arbres et le sol n'était pas toujours proprement entretenu. Au cœur de son processus de mythification, au milieu du $\mathrm{XX}^{\mathrm{e}}$ siècle, Yamada révèle le lien artificiellement construit entre ce jardin et la pensée métaphysique du bouddhisme zen, lien qui fait croire que le premier serait la représentation visuelle de la seconde. Par ailleurs, il remarque que l'identification du jardin de Ryōan-ji au zen est allée de pair avec celle de l'art japonais du tir à l'arc au zen, qui, quant à elle, s'est opérée avec l'ouvrage du professeur allemand de philosophie Eugen Herrigel, Zen in der Kunst des Bogenschiessens (1948). Cette trilogie du zen, du tir à l'arc et du jardin sec contribue à la fixation d'une image populaire de la culture japonaise comme culture hautement spirituelle et quelque peu mystérieuse. Une telle image s'est répandue aussi bien en Occident qu'au Japon pendant les années 1950 et 1960 et demeure encore populaire au début du XXI ${ }^{\text {e }}$ siècle, comme on peut le constater dans de nombreuses constructions de jardin secs, dont le plus emblématique est la réplique du jardin de Ryōan-ji à l'ambassade du Japon à Washington D.C. (1960), ainsi que dans la publication de divers livres intitulés suivant la formule "Zen and / in the Art of ..." , tels que Zen and the Art of Motorcycle Maintenance de Robert M. Pirsig (1974), qui mettent l'accent sur l'aspect métaphysique d'une pratique quotidienne ${ }^{3}$.

Les méthodes de Katahira et de Yamada marquent une rupture radicale avec les études formelles ou historiques d'un jardin particulier ou d'un style de jardin au Japon, en déplaçant le thème central au discours, au regard, à l'image, à l'interprétation et à la circulation d'idées à l'échelle internationale. Leur approche se nourrit, mais diffère, de celles de leurs prédécesseurs, tels que Wybe Kuitert, historien du jardin qui a remis en question, déjà dans sa thèse en 1988, l'image essentialiste et idéalisant du jardin japonais ${ }^{4}$, ou encore Makoto Suzuki, dont la thèse, en 1994, portait exclusivement sur le regard des étrangers sur les jardins japonais ${ }^{5}$. L'objectif de Kuitert était de proposer une compréhension plus sociohistorique de l'art du jardin, par un travail de recontextualisation des sources matérielles qui rétablit la réalité de la production des jardins dans le Japon historique, tandis que Katahira et Yamada s'éloignent de cette préoccupation historienne, suspendent la question de la véracité historique de l'art du jardin, et se focalisent sur l'analyse du regard et du mécanisme de la formation des idées, y compris des idées très déformées ou déformantes. Quant à Suzuki, malgré la liste impressionnante qu'il produit de textes en langues occidentales et d'images diffusées en Occident entre le XVI $\mathrm{X}^{\mathrm{e}}$ siècle et la fin 
du XXe siècle, son analyse est restée captive d'un paradigme dichotomique qui oppose les Japonais détenteurs du savoir-faire et de la sensibilité esthétique aux Occidentaux récepteurs, alors que les travaux de Katahira et de Yamada mettent en lumière le rôle des agents intermédiaires et la complexité des réactions de chaque acteur, ce qui les conduit à argumenter que la " japonité " de ces jardins est un produit des interactions internationales et n'en est pas une condition préalable.

\section{Rechercher des valeurs alternatives à celle de l'authenticité essentialiste}

La deuxième tendance récente des recherches sur le jardin japonais concerne les jardins qui existaient, ou existent encore, en dehors du Japon. Cela comprend des créations diverses : des parcs publics, des jardins privés, et de simples installations d'éléments horticoles, sculpturaux ou architecturaux qui sont évocateurs du Japon, tels que des arbres nains, des lanternes en pierre et des ponts arqués. Les plus anciens exemples sont les jardins créés lors des Expositions universelles de la deuxième moitié du XIX siècle et du début du $\mathrm{XX}^{\mathrm{e}}$ siècle. Ils accompagnaient les pavillons représentant la nation japonaise et servaient de lieu d'attraction à des fins à la fois diplomatique et commerciale. À ces installations temporaires ont succédé des jardins plus durables chez les riches propriétaires intéressés par la mode du japonisme. Certains d'entre eux ont survécu, et sont devenus publics, comme les jardins du banquier Albert Kahn à Boulogne-Billancourt, près de Paris. Après la Seconde Guerre mondiale beaucoup de jardins ont aussi été créés à l'occasion de la célébration d'une relation diplomatique ou d'un jumelage avec une ville japonaise, reflétant la croissance de la présence des Japonais sur la scène internationale. Longtemps considérés comme de médiocres imitations sans intérêt par rapport à leurs modèles au Japon, ces jardins commencent à attirer, depuis environ trois décennies, l'attention des chercheurs qui interrogent les valeurs du jardin japonais dans des contextes de création internationaux, des valeurs qui oscillent entre les critères d'authenticité essentialistes et les spécificités historiques et locales.

Les deux ouvrages de l'historien américain du jardin Kendall Brown, Japanese-Style Gardens of the Pacific West Coast et Quiet Beauty: The Japanese Gardens of North America, présentent respectivement vingt et vingt-six jardins japonais en Amérique du Nord ${ }^{6}$. Dans le texte introductif du premier ouvrage, Brown déclare qu'il considère ces lieux comme des " jardins authentiquement nord-américains ", car ils " nous racontent davantage sur l'Amérique et le Canada que sur le Japon [...]. Ces jardins, et leurs histoires, révèlent des aspects fondamentaux d'un siècle de relations politiques, économiques et culturelles de l'Amérique du Nord avec le Japon ${ }^{7}$. " Bien qu'il admette que la popularité de ces jardins repose sur l'idée qu'ils soient authentiquement japonais, il affirme que leur vraie valeur se trouve dans leur histoire, celle des désirs et des peurs de l'Occident vis-à-vis du Japon, de l'immigration japonaise aux États-Unis, et des interactions entre les deux.

Brown s'attarde par exemple à retracer les nombreux changements de gestionnaires et de morphologie du Japanese Tea Garden du Golden Gate Park à San Francisco (fig. 2), pour montrer à quel point il témoigne de l'évolution de la relation américano-japonaise et de celle de la perception américaine sur la culture japonaise. Le jardin fut initialement créé par l'entrepreneur George Turner Marsh pour l'Exposition internationale Midwinter de 1894, puis revendu après l'événement à la ville qui, vu son grand succès, souhaita le garder comme attraction commerciale. Sa gestion a alors été confiée à l'immigré japonais Makoto Hagiwara et à ses descendants qui l'ont entretenu jusqu'en mai 1942, lorsqu'ils sont déportés dans un camp d'Américano-Japonais créé quelques mois après le début 


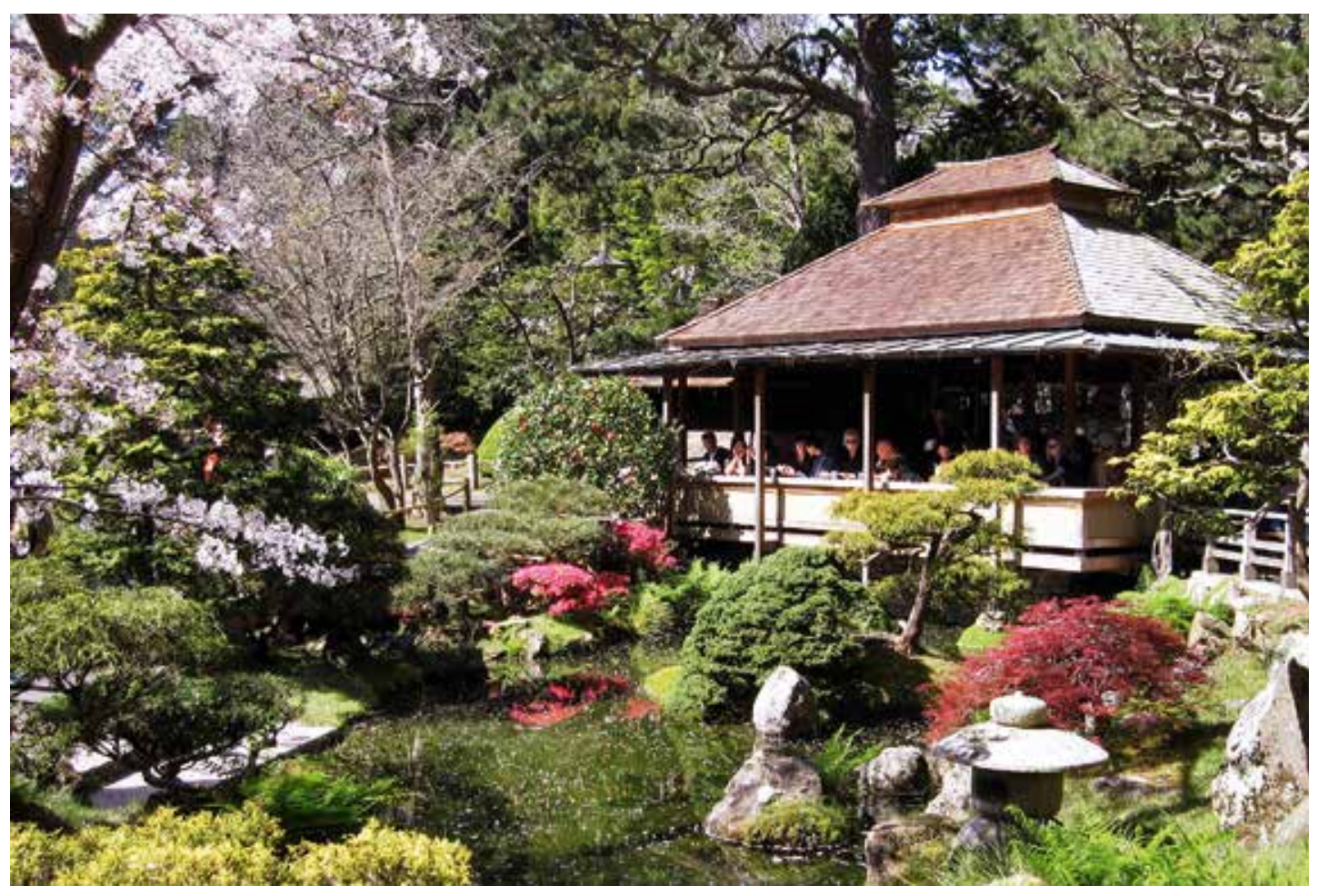

de la guerre du Pacifique. Hagiwara a assisté à l'agrandisse2. Japanese Tea Garden au Golden ment du jardin ainsi qu'au transfert d'une pagode et d'une Gate Park, San Francisco. porte de temple provenant de l'Exposition internationale Panama-Pacific de 1915, et à l'ajout d'un torii et d'un temple shintō - autant d'additions qui reflètent l'ampleur de cet engouement japonisant. En revanche, pendant la guerre, certains éléments, y compris le temple shintō et la maison des Hagiwara, ont été détruits, alors que la ville a continué à gérer le domaine sous le nom d'Oriental Tea Garden en embauchant des femmes chinoises. Les modifications et les ajouts se poursuivent après la guerre : le don d'une grande lanterne par le Consul général du Japon et la création d'un jardin sec par Nagao Sakurai en 1953, un an après la normalisation de la relation américano-japonaise, puis l'installation d'éléments commémoratifs pour la famille Hagiwara aux alentours de 1970, dans un climat de réhabilitation de la population d'origine japonaise.

Les jardins japonais créés dans les camps d'Américano-Japonais pendant la guerre du Pacifique font, eux aussi, l'objet de recherches depuis les années 1990, renouvelant la lecture de la fonction du jardin japonais à l'étranger. Si les autorités américaines ont construit ces camps dans des déserts de l'Ouest pour isoler la population d'ascendance japonaise de la côte ouest, quelques milliers de jardins, de tailles, de formes et de fonctions différentes, y ont été créés par des internés dont une partie importante était des professionnels du jardin. Anna Hosticka Tamura, dans son article « Gardens Below the Watchtower: Gardens and Meaning in World War II Japanese American Incarceration Camps $^{8}$ ", propose une interprétation de ces jardins comme symbole de résistance, à l'opposé de l'image plaisante du jardin japonais, commune aux États-Unis. Le Merritt Park du camp de Manzanar, par exemple, a été créé par un groupe de paysagistes, pépiniéristes et jardiniers, dirigé par Kuichiro Nishi, lui-même pépiniériste et emprisonné dans 


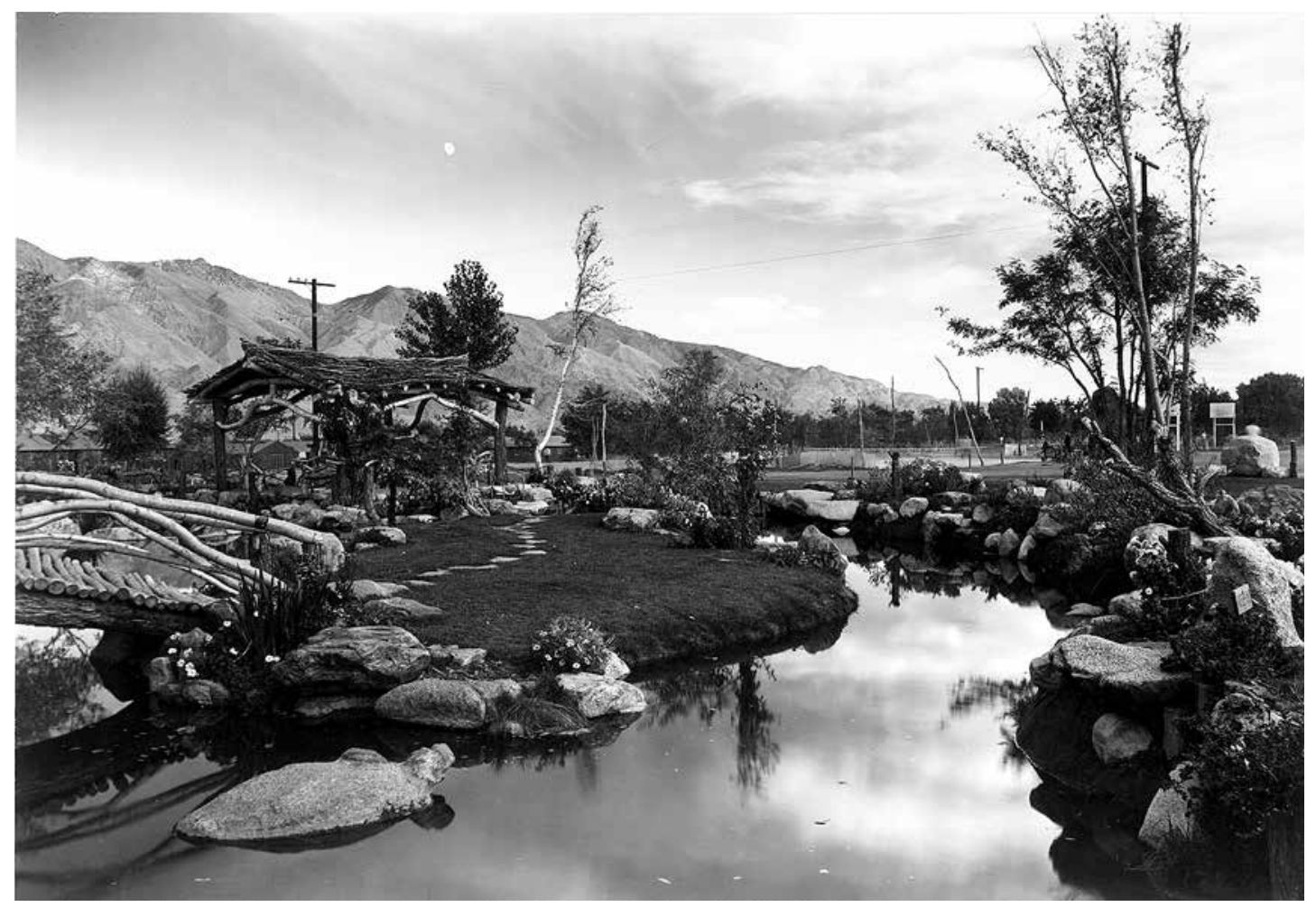

3. Ansel Adams, Merritt Park, site historique national de Manzanar (comté d'Inyo, Californie), 1943, Washington D.C., Library of Congress, Prints and Photographs Division, LOT 10479-2, nº 22. un camp d'ennemis étrangers avant de rejoindre sa famille à Manzanar. Avec leur savoir-faire et des matériaux importés ou trouvés sur place, ils ont construit un jardin d'agrément composé de cascades, de bassins, de ponts, d'une maison de thé ainsi que de plantes et de rochers soigneusement disposés, comme on peut le voir dans une photographie prise par Ansel Adams, montrant une pierre en forme de tortue, animal fréquemment représenté dans les jardins au Japon (fig. 3). Les jardins aux camps permettaient aux internés, selon Tamura, d'affronter le caractère insupportable de leur situation, à différents niveaux : la construction d'un jardin offrait la possibilité, pour les internés, de recréer une communauté par eux-mêmes, sur la base de leurs professions d'avant-guerre à partir d'une œuvre symbolisant leur culture ethnique ; elle était aussi un moyen de défier les limites imposées par l'autorité carcérale, car la construction implique de s'approprier un terrain et de négocier les permis de sortie temporaire pour aller chercher des matériaux. Les jardins ainsi créés tempéraient l'environnement étranger et inhospitalier des baraques au beau milieu du désert; de plus, le travail d'entretien au contact des plantes procurait des effets thérapeutiques, et permettait aussi de lutter contre l'inertie du confinement. En somme, les jardins japonais aidaient ces personnes déplacées à reprendre, certes, dans une infime mesure, le contrôle sur leurs conditions de vie.

En parallèle avec les recherches développées aux États-Unis, une vaste enquête collective sur les jardins japonais d'outre-mer a été menée au début des années 2000 par une commission créée au sein du Japanese Institue of Landscape Architecture et dirigée par Makoto Suzuki, historien du jardin cité plus haut. La commission, composée 
de trente-deux experts majoritairement japonais, incluant des universitaires, des concepteurs et des spécialistes de construction et d'entretien, a fait le recensement de plus de quatre cents jardins à travers le monde et s'est intéressée non seulement à leur histoire, mais surtout à leur état actuel et aux questions de leur construction, de leur entretien et de leur restauration. Cette enquête suit la recherche que Suzuki avait déjà entamée sur les cas historiques en Europe de l'Ouest et aux États-Unis ${ }^{9}$, et celle des organisations japonaises comme l'Urban Green Space Development Foundation qui veillaient sur ce phénomène alors qu'il prenait de l'ampleur pendant les deux dernières décennies du XXe siècle. Les membres de la commission ont constaté le besoin de réfléchir sur les perspectives d'avenir de ces jardins et sur celles des professionnels japonais qui étaient appelés à intervenir de plus en plus à l'étranger. Le résultat de leur enquête est présenté dans un rapport ainsi que sur des sites web spécialisés, avec des fiches détaillées de quatre-vingt jardins remarquables et des articles relatifs aux thématiques précitées ${ }^{10}$.

Ce qui est intéressant est que les contributeurs du rapport se montrent partagés entre, d'une part, l'envie de valoriser la spécificité locale de chaque jardin ainsi que la richesse d'échanges internationaux dont il témoigne et, d'autre part, l'embarras qu'ils ressentent face à l'éloignement de ces jardins par rapport au canon du jardin japonais tel qu'ils l'ont étudié tout au long de leur carrière et qu'ils sont censés représenter à l'occasion de leurs missions à l'étranger. Ils se demandent, par exemple, comment réagir s'ils sont sollicités dans le cadre d'un projet de restauration d'un jardin historique, créé initialement sur une image fantaisiste du Japon. Leurs hésitations et interrogations trahissent non seulement la complexité de la question de la "japonité ", mais aussi celle du jardin en tant qu'il est une création perpétuelle : composés de matières végétales, aquatiques et minérales dont l'évolution dépend du climat local et des interventions humaines sur une longue durée, les jardins changent d'apparence en permanence. Comme un jardin n'est jamais achevé au moment de son inauguration, la notion même d'auteur se brouille, entre les différentes instances du commanditaire, du concepteur, du constructeur, du jardinier, et peut-être même de la nature. Cela rend encore plus complexe la question de la " japonité " du jardin, à savoir si elle s'appuie sur l'ethnicité de son concepteur initial, sur celle d'un intervenant ultérieur, sur la provenance des matériaux qui le composent, ou bien sur la référence à laquelle renvoie l'image du Japon pour le propriétaire.

\section{Perspective d'avenir : des champs d'étude en friche}

Les recherches sur le jardin japonais en tant que phénomène international, qu'elles s'intéressent aussi bien à son image qu'à sa création, éclairent l'importance des échanges entre l'ici et l'ailleurs pour l'étude d'un art in situ. Elles remettent en question le lien intrinsèque entre l'homme et son environnement sur lequel s'appuie la japonité de l'art du jardin, et mettent en lumière le mécanisme de la transmission des informations, l'histoire du transfert des mains-d'œuvre, des matériaux et des savoir-faire, le rôle des différents acteurs et l'évolution de chaque jardin dans le temps; en d'autres termes, elles soulèvent des questions intéressantes pour réfléchir sur l'art dans le monde globalisé, qui se distinguent des questions concernant la circulation des objets d'art transportables et conservables dans les musées.

Ces recherches se développent majoritairement selon un axe Japon - Occident, et en particulier celui entre le Japon et les pays anglo-saxons tels que le Royaume-Uni et les États-Unis, comme on peut le constater dans les travaux que nous avons présentés et aussi dans l'ouvrage le plus récemment publié sur ce thème : Spaces in Translation: 
Japanese Gardens and the West, de Christian Tagsold, qui discute les jardins japonais des États-Unis et des différents pays d'Europe comme un ensemble plutôt monolithique ${ }^{11}$. Bien qu'elle reflète la densité des relations historiques entre le Japon et ces pays, cette tendance dépend aussi du flux d'information actuel, dominé par la documentation en anglais. Aussi, on peut se demander si les conclusions qui en sont tirées ne risquent pas de simplifier le phénomène international du jardin japonais, qui pourrait en réalité, dans le sens qui vient d'être évoqué, être encore plus complexe. En guise de conclusion, nous souhaitons présenter deux approches complémentaires possibles, aux marges de l'axe majeur entre le Japon et les pays anglo-saxons : la première en France et la deuxième en Asie orientale.

En France, le paysagiste Bernard Jeannel, qui a participé à la restauration du parc oriental de Maulévrier dans les années 1980, a publié en 1995 un ouvrage établissant une liste de vingt-neuf jardins japonais en France, mais sans encourager des recherches non essentialistes équivalentes à celles de Suzuki et de Brown ${ }^{12}$. Néanmoins, quelques études de cas suggèrent d'apprécier ces jardins franco-japonais comme des exemples de la prise de liberté artistique qui poussent le phénomène vers une autre direction. Le professeur de littérature française Junji Suzuki a enquêté sur le parcours de Wasuke Hata, un jardinier japonais arrivé en France à la fin du XIX $X^{e}$ siècle qui créa des jardins chez quelques amateurs japonisants de l'époque, tels que Robert de Montesquiou et Hugues Krafft. En découvrant l'intérêt de quelques personnalités du milieu littéraire parisien pour ce phénomène en marge du japonisme, Suzuki identifie une éventuelle source d'inspiration de Marcel Proust, dans son appréciation des objets horticoles japonais, pour le déploiement spectaculaire de l'espace et du temps à partir d'un objet minuscule ${ }^{13}$.

Wybe Kuitert, historien du jardin que nous avons cité plus haut, a analysé quant à lui la manière dont les aspirations postmodernes ont joué un rôle dans la conception du

4. Jardin japonais contemporain du musée départemental Albert-Kahn, Boulogne-Billancourt. jardin japonais contemporain que l'agence paysagiste Takano Landscape Planning a créé au musée Albert-Kahn à Boulogne-Billancourt à la fin des années 1980 (fig. 4) ${ }^{14}$.

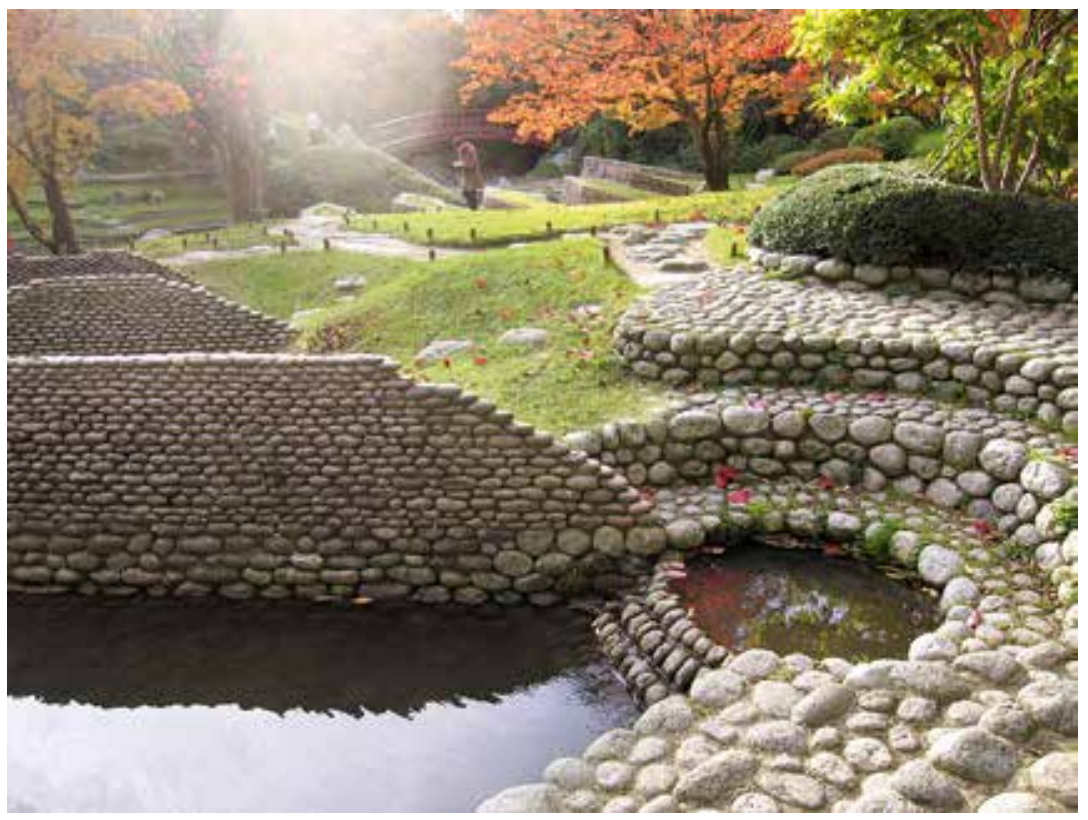


Ces cas franco-japonais montrent qu'il existe une possibilité d'élargir les études au-delà des jardins officiellement nommés japonais, et surtout aux autres domaines artistiques, pour étudier les rapports entre différents arts.

Les jardins japonais qui ont été créés dans les pays d'Asie orientale sont, quant à eux, très peu étudiés en comparaison avec les cas européens et nord-américains. Il est clair que la colonisation et les guerres provoquées par le Japon impérialiste ainsi que les relations d'après-guerre entre le Japon et ces pays ont eu un impact sur la façon dont ces jardins ont été construits, puis détruits ou abandonnés. Les recherches sur ces jardins n'ont débuté que tout récemment : Makoto Suzuki et ses collaborateurs ont présenté les résultats de leurs recherches sur la Corée du Sud en $2018^{15}$, et l'historien du jardin Takashi Awano prépare actuellement des articles sur les jardins créés par les colons japonais à Taïwan après son enquête de terrain en $2019^{16}$. Étant donné la situation coloniale de longue durée qui a entraîné l'installation massive d'une population japonaise et la japonisation de la population locale, il s'agit de s'intéresser non seulement aux cas particuliers des palais et des demeures de la classe gouvernante, mais aussi à la création de nombreux jardins plus ordinaires. Ces recherchent restent préliminaires : il s'agit d'établir une liste provisoire des jardins, de récolter des informations dans les archives locales pour reconstituer leurs histoires et leurs morphologies, avant de leur donner une signification. Nous espérons que ces études pourront ouvrir d'autres perspectives au-delà du schéma d'analyse post-orientaliste appliqué dans les cas d'étude entre le Japon et l'Occident. 


\section{Hiromi Matsugi}

Hiromi Matsugi est historienne de l'art et Assistant Professor à I'International Research Center for Japanese Studies, à Kyōto. Elle est auteure de I'article « Jardins japonais en France : exotisme, adaptation, invention ", dans Projets de paysage, $\mathrm{n}^{\circ}$ 6, juillet 2011.

\section{NOTES}

Tous les noms, y compris ceux d'origine japonaise, sont ici transcrits dans l'ordre prénom-patronyme. Ce choix découle de la particularité de l'article qui cite des personnes aux parcours internationaux divers. L'auteure de l'article remercie les relecteurs pour leurs expertises précieuses. Elle a intégré certaines de leurs suggestions et espère explorer les autres pistes dans ses travaux à venir.

1. Miyuki Katahira, Nihon teien zō no keisei 日本庭園 像の形成, Kyōto, Shibunkaku, 2014.

2. Shōji Yamada, Zen to yū na no nihonmaru 禅という 名の日本丸, Tōkyō, Kōbundō, 2005 ; Shots in the Dark: Japan, Zen, and the West, Earl Hartman (trad. angl.), Chicago / Londres / Kyōto, The University of Chicago Press / International Research Center for Japanese Studies, 2009.

3. Trente-cinq livres et articles intitulés de cette manière, publiés en anglais entre 1974 et 2003, sont cités par Yamada dans le premier chapitre de Yamada, 2005, cité n. 2.

4. Wybe Kuitert, Themes, Scenes, and Taste in the History of Japanese Garden Art, Amsterdam, J. C. Gieben, 1988, et sa réédition Themes in the History of Japanese Garden Art, Honolulu, University of Hawaï Press, 2002.

5. Makoto Suzuki, Ōbeijin no nihonteienkan 欧米人の 日本庭園観/ The Image and View of Japanese Gardens in the Minds of Westerners, Zōengaku ronshū [la revue de I'Université d'agriculture de Tōkyō], supplément $\mathrm{n}^{\circ} 2,1997$.

6. Kendall $\mathrm{H}$. Brown et Melba Levick (photographe), Japanese-Style Gardens of the Pacific West Coast, New York, Rizzoli, 1999 ; Kendall H. Brown et David M. Cobb (photographe), Quiet Beauty: The Japanese Gardens of North America, Tōkyō, Tuttle, 2013.

7. Brown et Levick, 1999, cité n. 6, p. 10 (traduction de l'auteure).

8. Anna Hosticka Tamura, "Gardens Below the Watchtower: Gardens and Meaning in World War II Japanese American Incarceration Camps ", dans Landscape Journal, no 23 (1), 2004, p. 1-21.

9. Suzuki, 1997, cité n. 5, p. 72-98.

10. Makoto Suzuki et al., "Kaigai no nihonteien" chōsa hōkokusho「海外の日本庭園」調査報告書 et sa version anglaise Japanese Gardens outside of Japan: Research Report, Tōkyō, Japanese Institute of Landscape
Architecture, respectivement 2006 et 2007. Les articles en anglais sont consultables en ligne, sur https://www. jila-zouen.org/journal/overseasjgardens, et les fiches de jardins sur http://www.nodaigarden.jp/.

11. Christian Tagsold, Spaces in Translation: Japanese Gardens and the West, Philadelphia, University of Pennsylvania Press, 2017.

12. Bernard Jeannel, Jardins japonais en France : art et poésie du paysage, Paris, Nathan, 1995.

13. Junji Suzuki, "Maruseru purūsto to nihon no "niwa" (Marcel Proust et deux "jardins" japonais) ", dans Revue de Hiyoshi. Langue et littérature françaises, no 12, 1991, p. 155-171. Idem, "Le jardinier japonais de Robert de Montesquiou - ses évocations dans les milieux littéraires ", dans Cahiers Edmond et Jules de Goncourt, $\mathrm{n}^{\circ} 18,2011$, p. 103-112.

14. Wybe Kuitert, "Discourse and Creation: Two Japanese Gardens to contemplate in Paris ", dans Shakkei, no 15 (1), été 2008, p. 18-24.

15. Makoto Suzuki et al., "Studies of the Japanese Gardens Created in South Korea: Historical Backgrounds and Significant Characteristics of the Remaining Japanese Gardens ", et Kwang-pyo Hong et al., "Studies of the Japanese Gardens Created in South Korea: Overall Conditions and Typological Classification of the remaining Japanese Gardens ", dans The 15th International Landscape Architectural Symposium of Japan, China and Korea: Landscape as Urban Infrastructure Program and Proceedings - Electronic version, Tōkyō, Japanese Institute of Landscape Architecture, 2016, p. 195-200, et p. 301-306.

16. Takashi Awano, "Nihon tōchi jidai no taiwan ni zōei sareta kantei, jūtaku, sekkyaku shisetsu no teien 日本統治時代の台湾に造営された官邸・住宅・接客 施設の庭園 》 [Les jardins dans les résidences officielles, les maisons et les établissements de plaisir à Taïwan de l'époque coloniale japonaise], dans Niwa, $n^{\circ} 238$, février 2020, p. 106-107. D'autres articles sont en cours de préparation. Je remercie Takashi Awano pour m'avoir donné l'accès à ses manuscrits. 